\title{
SPREADING SPEEDS AND TRAVELING WAVES FOR NON-MONOTONE INTEGRODIFFERENCE EQUATIONS
}

\author{
SZE-BI HSU* AND XIAO-QIANG $\mathrm{ZHAO}^{\dagger}$
}

\begin{abstract}
The spreading speeds and traveling waves are established for a class of non-monotone discrete-time integrodifference equation models. It is shown that the spreading speed is linearly determinate and coincides with the minimal wave speed of traveling waves.
\end{abstract}

Key words. Integrodifference equations, non-monotone integral operators, spreading speeds, linear determinacy, traveling waves.

AMS subject classifications. 37L15, 39A11, 92D25

1. Introduction. The invasion speed is a fundamental characteristic of biological invasions, since it describes the speed at which the geographic range of the population expands, see, e.g., $[6,8,9,15]$ and references therein. Aronson and Weinberger $[1,2]$ first introduced the concept of the asymptotic speed of spread (in short, spreading speed) for reaction-diffusion equations and showed that it coincides with the minimal wave speed for traveling waves under appropriate assumptions. Weinberger [20] and Lui [13] established the theory of spreading speeds and monostable traveling waves for monotone (order-preserving) operators. This theory has been greatly developed recently in $[21,10,11,12]$ to monotone semiflows so that it can be applied to various discrete- and continuous-time evolution equations admitting the comparison principle.

It is known that many discrete- and continuous-time population models with spatial structure are not monotone. For example, scalar discrete-time integrodifference equations with non-monotone growth functions, and predator-prey type reactiondiffusion systems are among such models. The spreading speeds were obtained for some non-monotone continuous-time integral equations and time-delayed reactiondiffusion models in $[17,19]$, and a general result on the nonexistence of traveling waves was also given in [19, Theorem 3.5]. The existence of monostable traveling waves were established for several classes of non-monotone time-delayed reactiondiffusion equations in $[22,4,16,14]$. For certain types of non-monotone discrete-time integrodifference equation models, non-monotone traveling waves and even traveling cycles were observed in [7] by numerical simulations. In [7, 9, 15, 8], the monotone linear systems, resulting from the linearization of the non-monotone discrete-time models at zero, were used to estimate spreading speeds. It is worthy to find sufficient conditions under which the spreading speed is linearly determinate for these non-monotone systems.

The purpose of our current paper is to study the spreading speeds and traveling waves for non-monotone discrete-time systems. As a starting point, we consider scalar integrodifference equations with non-monotone growth functions. The key techniques are to sandwich the given growth function in between two appropriate nondecreasing functions (for spreading speeds) and to construct a closed and convex subset in an

\footnotetext{
*Department of Mathematics, National Tsing Hua University, Hsinchu, Taiwan, R. O. China (sbhsu@math.nthu.edu.tw), supported in part by the National Council of Science of Republic of China.

${ }^{\dagger}$ Department of Mathematics and Statistics, Memorial University of Newfoundland, St. John's, NL A1C 5S7, Canada (xzhao@math.mun.ca), supported in part by the NSERC of Canada and the MITACS of Canada.
} 
appropriate Banach space (for traveling waves). Consequently, we obtain a set of sufficient conditions for the existence of the spreading speed, and the existence and nonexistence of traveling waves. It turns out that the spreading speed is linearly determinate and coincides with the minimal wave speed of traveling waves for this class of non-monotone discrete-time integrodifference equation population models.

The rest parts of this paper are organized as follows. In section 2 , we first present a general result for monotone integrodifference equations, then we establish the spreading speed $c^{*}$ by the comparison method and a fluctuation type argument. In section 3 , we use the Schauder fixed point theorem to obtain the existence of traveling waves with the wave speed $c>c^{*}$. The property of the spreading speed is employed to prove the asymptotic property of the wave profile at $+\infty$ and the nonexistence of traveling waves with $c<c^{*}$. A limiting argument gives the existence of the traveling wave with the wave speed $c^{*}$. Section 4 is aimed at the applications of the main results to three types of growth functions arising from population biology.

2. Spreading speeds. Let $\mathcal{C}$ be the space of all bounded and continuous functions from $\mathbb{R}$ to $\mathbb{R}$ equipped with the compact open topology. For a given number $r>0$, let $\mathcal{C}_{r}:=\{\phi \in \mathcal{C}: 0 \leq \phi(x) \leq r, \forall x \in \mathbb{R}\}$.

Let $k(x)$ be a nonnegative Lebesgue measurable function on $\mathbb{R}$. Throughout this paper, we assume that the kernel $k(x)$ has the following property:

(K) $\int_{\mathbb{R}} k(y) d y=1, k(-y)=k(y), \forall y \in \mathbb{R}$, and $\int_{\mathbb{R}} e^{-\alpha y} k(y) d y<\infty, \forall \alpha \in[0, \Delta)$, where $\Delta>0$ is the abscissa of convergence and it may be infinity.

We consider a discrete-time integrodifference equation

$$
u_{n+1}(x)=\int_{\mathbb{R}} h\left(u_{n}(y)\right) k(x-y) d y, x \in \mathbb{R}, n \geq 0
$$

with $u_{0} \in \mathcal{C}$. Assume that there exists $\beta>0$ such that

(H1) $h \in C([0, \beta],[0, \beta]), h(0)=0, h^{\prime}(0)>1, h(\beta)=\beta$, and there is $L_{0}>0$ such that $\left|h\left(u_{1}\right)-h\left(u_{2}\right)\right| \leq L_{0}\left|u_{1}-u_{2}\right|, \forall u_{1}, u_{2} \in[0, \beta]$.

(H2) $u<h(u) \leq h^{\prime}(0) u, \forall u \in(0, \beta)$, and $h(u)$ is nondecreasing in $u \in[0, \beta]$.

Let $U(x)$ be a continuous function on $\mathbb{R}$. We say $U(x+c n)$ is a traveling wave solution of (2.1) with the wave speed $c$ if $u_{n}(x)=U(x+c n), \forall n \geq 0$, satisfies (2.1), and $U(x+c n)$ connects 0 to $\beta$ if $U(-\infty)=0$ and $U(+\infty)=\beta$. It is easy to see that $U(x+c n)$ is a traveling wave solution of $(2.1)$ if and only if

$$
U(\xi)=\int_{\mathbb{R}} h(U(\xi-c-y)) k(y) d y, \quad \forall \xi \in \mathbb{R} .
$$

Define

$$
c_{h}^{*}=\inf _{\mu \in(0, \Delta)} \frac{\ln \left(h^{\prime}(0) \int_{\mathbb{R}} e^{-\mu y} k(y) d y\right)}{\mu} .
$$

The following result is essentially due to Weinberger [20], and shows that $c_{h}^{*}$ is not only the spreading speed but also the minimal wave speed of monotone traveling waves for system (2.1).

THEOREM 2.1. Let (H1) and (H2) hold. Then the following statements are valid:

(i) For any $u_{0} \in \mathcal{C}_{\beta}$ with compact support, the solution of (2.1) satisfies

$$
\lim _{n \rightarrow \infty,|x| \geq c n} u_{n}(x)=0, \forall c>c_{h}^{*} .
$$


(ii) For any $u_{0} \in \mathcal{C}_{\beta} \backslash\{0\}$, the solution of (2.1) satisfies $\lim _{n \rightarrow \infty,|x| \leq c n} u_{n}(x)=$ $\beta, \forall c \in\left(0, c_{h}^{*}\right)$.

(iii) For any $c \geq c_{h}^{*}$, (2.1) has a traveling wave $U(x+c n)$ connecting 0 to $\beta$ such that $U(x)$ is nondecreasing in $x$, and for any $c \in\left(0, c_{h}^{*}\right)$, (2.1) has no traveling wave $U(x+c n)$ connecting 0 to $\beta$.

Proof. The existence of the spreading speed, together with the formula (2.2), and traveling waves is a straightforward consequence of [20, Theorems 6.1-6.6] in the case where $\Delta=+\infty$, and [11, Theorem 2.11 and Theorem 2.15] with $\tau=0$ in the case where $\Delta<+\infty$. Indeed, the proof of [11, Theorem 3.10] implies that [11, Theorem 3.10] with $\inf _{\mu>0} \Phi(\mu)$ replaced by $\inf _{0<\mu<\Delta} \Phi(\mu)$ is still valid, provided that (C5) holds for all $\mu_{1}, \mu_{2} \in(-\Delta, \Delta)$ and $\Phi(\mu)$ assumes its minimum value at $\mu^{*} \in(0, \Delta)$. Thus, the formula (2.2) also holds in the case where $\Delta<+\infty$. By [11, Theorem $3.5]$, it follows that the number $r=r_{\sigma}$ in [11, Theorem 2.15] can be chosen to be independent of $\sigma>0$. For any $u_{0} \in \mathcal{C}_{\beta} \backslash\{0\}$, it is easy to show that there exists an integer $n_{0} \geq 1$ such that $u_{n_{0}}(x)>0$ for $x$ in an interval of length greater $2 r$. Taking $u_{n_{0}}(x)$ as a new initial data, we see that conclusion (ii) holds. The nonexistence of traveling waves is implied by conclusion (ii) (see also [11, Theorem 4.1]).

Next we consider the discrete-time integrodifference equation

$$
u_{n+1}(x)=\int_{\mathbb{R}} f\left(u_{n}(y)\right) k(x-y) d y, \quad x \in \mathbb{R}, n \geq 0
$$

with $u_{0} \in \mathcal{C}$. Assume that there exists $b>0$ such that

(F1) $f \in C([0, b],[0, b]), f(0)=0, f^{\prime}(0)>1$, and there is $L>0$ such that $\mid f\left(u_{1}\right)-$ $f\left(u_{2}\right)|\leq L| u_{1}-u_{2} \mid, \forall u_{1}, u_{2} \in[0, b]$.

(F2) $f(u) \leq f^{\prime}(0) u, \forall u \in[0, b]$, and there is $u^{*} \in(0, b]$ such that $f\left(u^{*}\right)=u^{*}$, $f(u)>u, \forall u \in\left(0, u^{*}\right)$, and $0<f(u)<u, \forall u \in\left(u^{*}, b\right]$.

Define

$$
f^{+}(u)=\max _{0 \leq v \leq u} f(v), \quad f^{-}(u)=\min _{u \leq v \leq b} f(v), \quad \forall u \in[0, b] .
$$

It then follows that

$$
f^{-}(u) \leq f(u) \leq f^{+}(u), \forall u \in[0, b],
$$

that both $f^{+}$and $f^{-}$are nondecreasing and Lipschitz continuous, with the Lipschitz constant $L$, on $[0, b]$, and that there exists $\delta_{0} \in(0, b]$ such that $f^{ \pm}(u)=f(u), \forall u \in$ $\left[0, \delta_{0}\right]$. Let $u_{ \pm}^{*}$ be such that $f^{ \pm}\left(u_{ \pm}^{*}\right)=u_{ \pm}^{*}$. Then $0<u_{-}^{*} \leq u^{*} \leq u_{+}^{*} \leq b$.

To obtain the upward convergence as stated in Theorem 2.1 (ii), we need to impose one of the following two additional conditions on $f$.

(C1) $u^{*}=b$ and $f(u)$ is nondecreasing in $u \in\left[b-\epsilon_{0}, b\right]$ for some $\epsilon_{0} \in(0, b)$.

(C2) $\frac{f(u)}{u}$ is strictly decreasing for $u \in(0, b]$, and $f(u)$ has the property (P) that for any $v, w \in(0, b]$ satisfying $v \leq u^{*} \leq w, v \geq f(w)$ and $w \leq f(v)$, we have $v=w$.

Motivated by the proofs of [17, Theorems 2.9 and 2.12], we have the following observation.

LEMma 2.2. Either of the following two conditions is sufficient for the property (P) in condition (C2) to hold:

(P1) uf(u) is strictly increasing for $u \in(0, b]$.

(P2) $f(u)$ is nonincreasing for $u \in\left[u^{*}, b\right]$, and $\frac{f^{2}(u)}{u}$ is strictly decreasing for $u \in$ $\left(0, u^{*}\right]$. 
Proof. Let $v, w \in(0, b]$ be given such that $v \leq u^{*} \leq w, v \geq f(w)$ and $w \leq f(v)$. In the case where (P1) holds, since $v f(v) \geq f(w) f(v) \geq w f(w)$, it follows that $v \geq w$, and hence $v=w$. In the case where (P2) holds, we have $v \geq f(w) \geq f(f(v))=f^{2}(v)$, and hence, $\frac{f^{2}(v)}{v} \leq 1=\frac{f^{2}\left(u^{*}\right)}{u^{*}}$. It then follows that $v \geq u^{*}$, and hence, $v=u^{*}$. Since $u^{*} \leq w \leq f(v)=f\left(u^{*}\right)=u^{*}$, we further have $w=u^{*}=v$.

Now we are a position to prove the main result of this section.

THEOREM 2.3. Let (F1) and (F2) hold and $c_{f}^{*}$ be defined as in (2.2) with $h=f$. Then the following statements are valid:

(1) For any $u_{0} \in \mathcal{C}_{u_{+}^{*}}$ with compact support, the solution of (2.3) satisfies $\lim _{n \rightarrow \infty,|x| \geq c n} u_{n}(x)=0, \forall c>c_{f}^{*}$.

(2) For any $u_{0} \in \mathcal{C}_{u_{+}^{*}} \backslash\{0\}$, the solution of (2.3) satisfies

$$
u_{-}^{*} \leq \liminf _{n \rightarrow \infty,|x| \leq c n} u_{n}(x) \leq \limsup _{n \rightarrow \infty,|x| \leq c n} u_{n}(x) \leq u_{+}^{*}, \forall c \in\left(0, c_{f}^{*}\right) .
$$

(3) If, in addition, either (C1) or (C2) holds, then for any $u_{0} \in \mathcal{C}_{u_{+}^{*}} \backslash\{0\}$, the solution of (2.3) satisfies $\lim _{n \rightarrow \infty,|x| \leq c n} u_{n}(x)=u^{*}, \forall c \in\left(0, c_{f}^{*}\right)$.

Proof. For convenience, let $c^{*}=c_{f}^{*}$. Define

$$
Q(\phi)(x)=\int_{\mathbb{R}} f(\phi(x-y)) k(y) d y, \quad Q^{ \pm}(\phi)(x)=\int_{\mathbb{R}} f^{ \pm}(\phi(x-y)) k(y) d y .
$$

Clearly, $Q^{ \pm}$is monotone (order preserving) on $\mathcal{C}_{b}$ and

$$
Q^{-}(\phi) \leq Q(\phi) \leq Q^{+}(\phi), \quad \forall \phi \in \mathcal{C}_{b}
$$

By Theorem 2.1, it follows that $c^{*}$ is the spreading speed for the discrete-time system $u_{n+1}=Q^{ \pm}\left(u_{n}\right)$ on $\mathcal{C}_{u_{ \pm}^{*}}$

Case 1. For a given $\phi \in \mathcal{C}_{u_{+}^{*}}$ with compact support, let $u_{n}=Q^{n}(\phi), u_{n}^{+}=$ $\left(Q^{+}\right)^{n}(\phi), \forall n \geq 0$. By the comparison principle (see, e.g., [20, Proposition 4.1]), we have

$$
0 \leq u_{n}(x) \leq u_{n}^{+}(x), \quad \forall x \in \mathbb{R}, n \geq 0
$$

For any $c>c^{*}$, Theorem 2.1 (i) implies that $\lim _{n \rightarrow \infty,|x| \geq c n} u_{n}^{+}(x)=0$, and hence $\lim _{n \rightarrow \infty,|x| \geq c n} u_{n}(x)=0$.

Case 2. For a given $\phi \in \mathcal{C}_{u_{+}^{*}} \backslash\{0\}$, define $\psi(x)=\min \left(\phi(x), u_{-}^{*}\right)$. Then $\psi \in$ $\mathcal{C}_{u_{-}^{*}} \backslash\{0\}$. Let $u_{n}^{-}=\left(Q^{-}\right)^{n}(\psi), \forall n \geq 0$. Since $\psi \leq \phi$, it follows from the comparison principle that

$$
0 \leq u_{n}^{-}(x) \leq u_{n}(x) \leq u_{n}^{+}(x), \quad \forall x \in \mathbb{R}, n \geq 0
$$

For any $c \in\left(0, c^{*}\right)$, Theorem 2.1 (ii) implies that $\lim _{n \rightarrow \infty,|x| \leq c n} u_{n}^{ \pm}(x)=u_{ \pm}^{*}$. Thus, we have

$$
u_{-}^{*} \leq \liminf _{n \rightarrow \infty,|x| \leq c n} u_{n}(x) \leq \limsup _{n \rightarrow \infty,|x| \leq c n} u_{n}(x) \leq u_{+}^{*}
$$

Case 3. In the case where (C1) holds, we see that $u^{*} \leq u_{+}^{*} \leq b=u^{*}$. Further, it follows from the definition of $f^{-}$that $f^{-}(u)=f(u), \forall u \in\left[b-\epsilon_{0}, b\right]$, and hence, 
$u_{-}^{*}=u^{*}$. Since $u_{-}^{*}=u_{+}^{*}=u^{*}$, the upward convergence in case (3) follows from the conclusion in case (2).

In the case where $(\mathrm{C} 2)$ holds, we use similar arguments as in the proof of [17, Lemma 3.10] (see also the proof of $\left[19\right.$, Theorem 2.5]). For any $(v, w) \in[0, b]^{2}$, let

$$
g(v, w)= \begin{cases}\min \{f(u): v \leq u \leq w\}, & \text { if } v \leq w, \\ \max \{f(u): w \leq u \leq v\}, & \text { if } w \leq v .\end{cases}
$$

Then $g(v, w)$ is nondecreasing in $v \in[0, b]$ and nonincreasing in $w \in[0, b]$. Moreover, $f(u)=g(u, u)$, and $g(v, w)$ is continuous in $(v, w) \in[0, b]^{2}$ (see [18, Section 2]). For a given $u_{0} \in \mathcal{C}_{u_{+}^{*}} \backslash\{0\}$, let $u_{n}=Q^{n}\left(u_{0}\right), \forall n \geq 0$. Then we have

$$
u_{n+1}(x)=\int_{\mathbb{R}} g\left(u_{n}(x-y), u_{n}(x-y)\right) k(y) d y, \quad n \geq 0 .
$$

For any $\beta \in\left(0, c^{*}\right)$, we define

$$
U_{*}(\beta):=\liminf _{n \rightarrow \infty,|x| \leq \beta n} u_{n}(x), \quad U^{*}(\beta):=\limsup _{n \rightarrow \infty,|x| \leq \beta n} u_{n}(x) .
$$

Let $c \in\left(0, c^{*}\right)$ be given. We fix a number $\gamma \in\left(c, c^{*}\right)$ and define

$$
V_{*}(c, \gamma)=\inf _{c<\beta<\gamma} U_{*}(\beta), \quad V^{*}(c, \gamma)=\sup _{c<\beta<\gamma} U^{*}(\beta) .
$$

It then follows that

$$
V_{*}(c, \gamma) \leq U_{*}(\beta) \leq U^{*}(\beta) \leq V^{*}(c, \gamma), \quad \forall \beta \in[c, \gamma] .
$$

By the conclusion in case 2, we have

$$
0<u_{-}^{*} \leq U_{*}(\beta) \leq U^{*}(\beta) \leq u_{+}^{*}, \quad \forall \beta \in\left(0, c^{*}\right),
$$

and hence,

$$
0<u_{-}^{*} \leq V_{*}(c, \gamma) \leq V^{*}(c, \gamma) \leq u_{+}^{*} \leq b .
$$

For any $\beta \in(c, \gamma)$, we choose two sequences $n_{j} \rightarrow \infty$, and $x_{j} \in \mathbb{R}$ with $\left|x_{j}\right| \leq \beta n_{j}$ such that $\lim _{j \rightarrow \infty} u_{n_{j}}\left(x_{j}\right)=U_{*}(\beta)$. It is easy to see that

$$
U_{*}(\gamma) \leq \liminf _{j \rightarrow \infty} u_{n_{j}-1}\left(x_{j}-y\right) \leq \limsup _{j \rightarrow \infty} u_{n_{j}-1}\left(x_{j}-y\right) \leq U^{*}(\gamma), \forall y \in \mathbb{R}
$$

Since $u_{n_{j}}\left(x_{j}\right)=\int_{\mathbb{R}} g\left(u_{n_{j}-1}\left(x_{j}-y\right), u_{n_{j}-1}\left(x_{j}-y\right)\right) k(y) d y$, it follows from Fatou's lemma that

$$
U_{*}(\beta) \geq \int_{\mathbb{R}} \liminf _{j \rightarrow \infty} g\left(u_{n_{j}-1}\left(x_{j}-y\right), u_{n_{j}-1}\left(x_{j}-y\right)\right) k(y) d y,
$$

and hence

$$
\begin{aligned}
U_{*}(\beta) & \geq \int_{\mathbb{R}} g\left(U_{*}(\gamma), U^{*}(\gamma)\right) k(y) d y \\
& =g\left(U_{*}(\gamma), U^{*}(\gamma)\right) \geq g\left(V_{*}(c, \gamma), V^{*}(c, \gamma)\right) .
\end{aligned}
$$


Similarly, we have

$$
U^{*}(\beta) \leq g\left(U^{*}(\gamma), U_{*}(\gamma)\right) \leq g\left(V^{*}(c, \gamma), V_{*}(c, \gamma)\right) .
$$

Thus,

$$
V_{*}(c, \gamma) \geq g\left(V_{*}(c, \gamma), V^{*}(c, \gamma)\right), \quad V^{*}(c, \gamma) \leq g\left(V^{*}(c, \gamma), V_{*}(c, \gamma)\right) .
$$

By the definition of function $g$, we can find $v, w \in\left[V_{*}(c, \gamma), V^{*}(c, \gamma)\right] \subset(0, b]$ such that

$$
g\left(V^{*}(c, \gamma), V_{*}(c, \gamma)\right)=f(v) \quad \text { and } \quad g\left(V_{*}(c, \gamma), V^{*}(c, \gamma)\right)=f(w) .
$$

It then follows from (2.5) that

$$
f(w) \leq V_{*}(c, \gamma) \leq v, w \leq V^{*}(c, \gamma) \leq f(v),
$$

and hence,

$$
\frac{f(w)}{w} \leq 1=\frac{f\left(u^{*}\right)}{u^{*}} \leq \frac{f(v)}{v}
$$

This, together with the strict monotonicity of $\frac{f(u)}{u}$ on $(0, b]$, implies that $v \leq u^{*} \leq w$. By (2.6) and the property (P), we obtain $v=w$. It then follows from (2.6) that $V_{*}(c, \gamma) \geq f(w)=f(v) \geq V^{*}(c, \gamma)$, and hence, $0<V_{*}(c, \gamma)=V^{*}(c, \gamma) \leq b$. From (2.5), we have

$$
V_{*}(c, \gamma) \geq g\left(V_{*}(c, \gamma), V_{*}(c, \gamma)\right) \geq V^{*}(c, \gamma),
$$

and hence, $0<V_{*}(c, \gamma)=f\left(V_{*}(c, \gamma)\right)$. By the uniqueness of positive fixed point of $f$ in $[0, b]$, it follows that $V_{*}(c, \gamma)=u^{*}$. Consequently,

$$
u^{*}=V_{*}(c, \gamma) \leq U_{*}(c) \leq U^{*}(c) \leq V^{*}(c, \gamma)=u^{*},
$$

which implies that $\lim _{n \rightarrow \infty,|x| \leq c n} u_{n}(x)=u^{*}$ for any $c \in\left(0, c^{*}\right)$.

REMARK 2.1. Under the assumption that $\int_{\mathbb{R}^{m}} k(y) d y=1, k(x)=k(y), \forall x, y \in$ $\mathbb{R}^{m}$ with $|x|=|y|$, Theorem 2.3 is still valid if we replace $\int_{\mathbb{R}}$ with $\int_{\mathbb{R}^{m}}$.

3. Traveling waves. In this section, we establish the existence and nonexistence of traveling waves for systems (2.3) by appealing to the Schauder fixed point theorem and the property of the spreading speed.

For a given $\lambda>0$, let

$$
X_{\lambda}:=\left\{\phi \in C(\mathbb{R}, \mathbb{R}): \quad \sup _{\xi \in \mathbb{R}}|\phi(\xi)| e^{-\lambda \xi}<+\infty\right\}
$$

and $\|\phi\|_{\lambda}=\sup _{\xi \in \mathbb{R}}|\phi(\xi)| e^{-\lambda \xi}$. It then follows that $\left(X_{\lambda},\|\cdot\|_{\lambda}\right)$ is a Banach space.

Define

$$
\Phi(\lambda)=\frac{\ln \left(f^{\prime}(0) \int_{\mathbb{R}} e^{-\lambda y} k(y) d y\right)}{\lambda}, \quad \forall \lambda \in(0, \Delta)
$$

and

$$
K(c, \lambda)=f^{\prime}(0) e^{-c \lambda} \int_{\mathbb{R}} e^{-\lambda y} k(y) d y, \quad \forall c \in \mathbb{R}_{+}, \lambda \in(0, \Delta) .
$$


By [11, Lemma 3.8], it follows that for any $c>c_{f}^{*}$, there exist $0<\lambda_{1}=\lambda_{1}(c)<\lambda_{2}=$ $\lambda_{2}(c)<\Delta$ such that $\Phi\left(\lambda_{1}\right)=c$ and $\Phi(\lambda)<c, \forall \lambda \in\left(\lambda_{1}, \lambda_{2}\right)$. Thus, we have

$$
K\left(c, \lambda_{1}\right)=1, \quad K(c, \lambda)<1, \forall \lambda \in\left(\lambda_{1}, \lambda_{2}\right) .
$$

Note that if $f^{\prime \prime}(0)$ exists, then $f(u) \geq f^{\prime}(0) u-a u^{2}, \forall u \in[0, \delta]$, for appropriate $a>0$ and $\delta>0$. To obtain the existence of traveling waves, we impose the following weaker condition on $f$ (cf. [3]).

(F3) There exist real numbers $\delta^{*} \in\left(0, \delta_{0}\right], \sigma>1$ and $a>0$ such that $f(u) \geq$ $f^{\prime}(0) u-a u^{\sigma}, \forall u \in\left[0, \delta^{*}\right]$.

TheOREM 3.1. Let $(F 1)-(F 3)$ hold. Then the following statements are valid:

(1) For any $c \in\left(0, c_{f}^{*}\right)$, (2.3) has no traveling wave $U(x+c n)$ with $U \in \mathcal{C}_{u_{+}^{*}} \backslash\{0\}$ and $U(-\infty)=0$.

(2) For any $c>c_{f}^{*}$, (2.3) has a traveling wave $U(x+c n)$ such that $U \in \mathcal{C}_{u_{+}^{*}} \backslash\{0\}$, $U(-\infty)=0$ and

$$
u_{-}^{*} \leq \liminf _{\xi \rightarrow+\infty} U(\xi) \leq \limsup _{\xi \rightarrow+\infty} U(\xi) \leq u_{+}^{*} .
$$

If, in addition, either (C1) or (C2) holds, then $U(+\infty)=u^{*}$.

Proof. Case 1. Assume, by contradiction, that for some $c_{0} \in\left(0, c_{f}^{*}\right),(2.3)$ has a traveling wave $u_{n}(x):=U\left(x+c_{0} n\right)$ with $U \in \mathcal{C}_{u_{+}^{*}} \backslash\{0\}$ and $U(-\infty)=0$. By Theorem $2.3(2)$, there holds

$$
\liminf _{n \rightarrow \infty,|x| \leq c n} u_{n}(x) \geq u_{-}^{*}>0, \quad \forall c \in\left(0, c_{f}^{*}\right) .
$$

Choose $\tilde{c} \in\left(c_{0}, c_{f}^{*}\right)$ and let $x=-\tilde{c} n$. Then $\liminf _{n \rightarrow \infty} u_{n}(-\tilde{c} n)=\liminf _{n \rightarrow \infty} U\left(\left(c_{0}-\tilde{c}\right) n\right)>0$, but $\lim _{n \rightarrow \infty} U\left(\left(c_{0}-\tilde{c}\right) n\right)=U(-\infty)=0$, a contradiction.

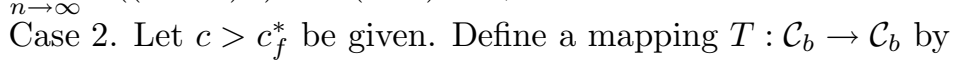

$$
T(\phi)(\xi)=\int_{\mathbb{R}} f(\phi(\xi-c-y)) k(y) d y, \quad \forall \xi \in \mathbb{R}, \phi \in \mathcal{C}_{b} .
$$

Let $T^{ \pm}$be defined as in (3.1) with $f$ replaced by $f^{ \pm}$. It then follows that $T^{ \pm}$is nondecreasing with respect to the pointwise ordering on $\mathcal{C}_{b}$, and that

$$
T^{-}(\phi) \leq T(\phi) \leq T^{+}(\phi), \forall \phi \in \mathcal{C}_{b} .
$$

Following [3], we define

$$
\phi^{+}(\xi):=\min \left\{u_{+}^{*} e^{\lambda_{1} \xi}, u_{+}^{*}\right\}, \quad \forall \xi \in \mathbb{R} .
$$

Since $f^{+}(u)$ is nondecreasing in $u$ and $\phi^{+}(\xi) \leq u_{+}^{*}, \forall \xi \in \mathbb{R}$, we obtain

$$
T^{+}\left(\phi^{+}\right)(\xi) \leq \int_{\mathbb{R}} f^{+}\left(u_{+}^{*}\right) k(y) d y=f^{+}\left(u_{+}^{*}\right)=u_{+}^{*}, \quad \forall \xi \in \mathbb{R} .
$$

Note that $f^{+}(u) \leq f^{\prime}(0) u, \forall u \in[0, b]$, and $\phi^{+}(\xi) \leq u_{+}^{*} e^{\lambda_{1} \xi}, \forall \xi \in \mathbb{R}$. Thus, we further have

$$
\begin{aligned}
T^{+}\left(\phi^{+}\right)(\xi) & \leq \int_{\mathbb{R}} f^{\prime}(0) \phi^{+}(\xi-c-y) k(y) d y \\
& \leq f^{\prime}(0) \int_{\mathbb{R}} u_{+}^{*} e^{\lambda_{1}(\xi-c-y)} k(y) d y \\
& =u_{+}^{*} e^{\lambda_{1} \xi} K\left(c, \lambda_{1}\right)=u_{+}^{*} e^{\lambda_{1} \xi}, \forall \xi \in \mathbb{R} .
\end{aligned}
$$


By (3.2), (3.3) and the definition of $\phi^{+}$, it then follows that $T^{+}\left(\phi^{+}\right) \leq \phi^{+}$. We first fix a sufficiently small $\epsilon^{*} \in\left(0, \lambda_{1}(\sigma-1)\right]$ such that $\lambda_{1}+\epsilon^{*}<\lambda_{2}$, and hence $K\left(c, \lambda_{1}+\epsilon^{*}\right)<1$. We then choose a sufficiently large number $M \geq 1$ such that

$$
\left(1+\frac{a\left(u_{+}^{*}\right)^{\sigma}}{f^{\prime}(0) \delta^{*} M}\right) K\left(c, \lambda_{1}+\epsilon^{*}\right)<1
$$

Following [19], we define

$$
\phi^{-}(\xi):=\max \left\{0, \delta^{*}\left(1-M e^{\epsilon^{*} \xi}\right) e^{\lambda_{1} \xi}\right\}, \quad \forall \xi \in \mathbb{R} .
$$

Let $\xi_{0}:=-\frac{\ln M}{\epsilon^{*}}$. Then we have

$$
\phi^{-}(\xi)=0, \forall \xi \geq \xi_{0}, \quad \phi^{-}(\xi)=\delta^{*} e^{\lambda_{1} \xi}-\delta^{*} M e^{\left(\lambda_{1}+\epsilon^{*}\right) \xi}, \forall \xi \leq \xi_{0} .
$$

Since $\delta^{*} \leq u_{+}^{*}, \xi_{0} \leq 0$ and $0<\epsilon^{*} \leq \lambda_{1}(\sigma-1)$, it is easy to see that

$$
0 \leq \phi^{-}(\xi) \leq \phi^{+}(\xi), \quad\left(\phi^{-}(\xi)\right)^{\sigma} \leq\left(u_{+}^{*}\right)^{\sigma} e^{\left(\lambda_{1}+\epsilon^{*}\right) \xi}, \quad \forall \xi \in \mathbb{R} .
$$

Clearly, we have

$$
T^{-}\left(\phi^{-}\right)(\xi) \geq 0, \quad \forall \xi \in \mathbb{R} .
$$

Since $\phi^{-}(\xi) \geq \delta^{*} e^{\lambda_{1} \xi}-\delta^{*} M e^{\left(\lambda_{1}+\epsilon^{*}\right) \xi}, \forall \xi \in \mathbb{R}$, it follows from (3.4) that

$$
\begin{aligned}
f^{\prime}(0) \phi^{-}(\xi)-a\left(\phi^{-}(\xi)\right)^{\sigma} & \geq f^{\prime}(0) \delta^{*} e^{\lambda_{1} \xi}-f^{\prime}(0) \delta^{*} M e^{\left(\lambda_{1}+\epsilon^{*}\right) \xi}-a\left(u_{+}^{*}\right)^{\sigma} e^{\left(\lambda_{1}+\epsilon^{*}\right) \xi} \\
& =f^{\prime}(0) \delta^{*} e^{\lambda_{1} \xi}-f^{\prime}(0) \delta^{*} M e^{\left(\lambda_{1}+\epsilon^{*}\right) \xi}\left(1+\frac{a\left(u_{+}^{*}\right)^{\sigma}}{f^{\prime}(0) \delta^{*} M}\right) \\
& \geq f^{\prime}(0) \delta^{*} e^{\lambda_{1} \xi}-\frac{f^{\prime}(0) \delta^{*} M}{K\left(c, \lambda_{1}+\epsilon^{*}\right)} e^{\left(\lambda_{1}+\epsilon^{*}\right) \xi}, \quad \forall \xi \in \mathbb{R} .
\end{aligned}
$$

In view of (F3) and the fact that $f^{-}(u)=f(u), \forall u \in\left[0, \delta_{0}\right]$, we then have

$$
\begin{aligned}
T^{-}\left(\phi^{-}\right)(\xi) & \geq \int_{\mathbb{R}}\left(f^{\prime}(0) \phi^{-}(\xi-c-y)-a\left(\phi^{-}(\xi-c-y)\right)^{\sigma}\right) k(y) d y \\
& \geq \int_{\mathbb{R}}\left(f^{\prime}(0) \delta^{*} e^{\lambda_{1}(\xi-c-y)}-\frac{f^{\prime}(0) \delta^{*} M}{K\left(c, \lambda_{1}+\epsilon^{*}\right)} e^{\left(\lambda_{1}+\epsilon^{*}\right)(\xi-c-y)}\right) k(y) d y \\
& =\delta^{*} e^{\lambda_{1} \xi} K\left(c, \lambda_{1}\right)-\frac{\delta^{*} M}{K\left(c, \lambda_{1}+\epsilon^{*}\right)} e^{\left(\lambda_{1}+\epsilon^{*}\right) \xi} K\left(c, \lambda_{1}+\epsilon^{*}\right) \\
& =\delta^{*} e^{\lambda_{1} \xi}-\delta^{*} M e^{\left(\lambda_{1}+\epsilon^{*}\right) \xi}, \quad \forall \xi \in \mathbb{R} .
\end{aligned}
$$

By (3.5), (3.6) and the definition of $\phi^{-}$, it follows that $T^{-}\left(\phi^{-}\right) \geq \phi^{-}$.

Now we fix a number $\lambda \in\left(0, \lambda_{1}\right)$. It is easy to see that both $\phi^{-}$and $\phi^{+}$are elements in $X_{\lambda}$. Thus, the set

$$
Y:=\left\{\phi \in X_{\lambda}: \phi^{-}(\xi) \leq \phi(\xi) \leq \phi^{+}(\xi), \forall \xi \in \mathbb{R}\right\}
$$

is a nonempty, closed and convex subset of $X_{\lambda}$. For any $\phi \in Y$, we have

$$
\phi^{-} \leq T^{-}\left(\phi^{-}\right) \leq T^{-}(\phi) \leq T(\phi) \leq T^{+}(\phi) \leq T^{+}\left(\phi^{+}\right) \leq \phi^{+},
$$


and hence $T(Y) \subset Y$. For any $\phi, \psi \in Y$, there holds

$$
\begin{aligned}
\|T(\phi)-T(\psi)\|_{\lambda} & =\sup _{\xi \in \mathbb{R}}|T(\phi)(\xi)-T(\psi)(\xi)| e^{-\lambda \xi} \\
& \leq L \cdot \sup _{\xi \in \mathbb{R}} \int_{\mathbb{R}}|\phi(\xi-c-y)-\psi(\xi-c-y)| e^{-\lambda \xi} k(y) d y \\
& \leq L\|\phi-\psi\|_{\lambda} \int_{\mathbb{R}} e^{-\lambda(c+y)} k(y) d y \\
& =\left(L e^{-\lambda c} \int_{\mathbb{R}} e^{-\lambda y} k(y) d y\right)\|\phi-\psi\|_{\lambda} .
\end{aligned}
$$

This implies that $T: Y \rightarrow Y$ is continuous. We further show that $T(Y)$ is precompact in $X_{\lambda}$. For any $\phi \in Y, \xi_{1}, \xi_{2} \in \mathbb{R}$, we have

$$
\begin{aligned}
\left|T(\phi)\left(\xi_{1}\right)-T(\phi)\left(\xi_{2}\right)\right| & =\left|\int_{\mathbb{R}} f(\phi(z))\left(k\left(\xi_{1}-c-z\right)-k\left(\xi_{2}-c-z\right)\right) d z\right| \\
& \leq b \int_{\mathbb{R}}\left|k\left(\xi_{1}-c-z\right)-k\left(\xi_{2}-c-z\right)\right| d z \\
& =b \int_{\mathbb{R}}\left|k\left(\xi_{1}-\xi_{2}+y\right)-k(y)\right| d y \\
& =b \cdot g\left(\xi_{1}-\xi_{2}\right),
\end{aligned}
$$

where $g(\xi)=\int_{\mathbb{R}}|k(\xi+y)-k(y)| d y, \forall \xi \in \mathbb{R}$. Since $\lim _{\xi \rightarrow 0} g(\xi)=0$, it follows that the family of functions $\{T(\phi)(\xi): \phi \in Y\}$ is uniformly bounded and equicontinuous in $\xi \in \mathbb{R}$. Thus, for any given sequence $\left\{\psi_{n}\right\}_{n \geq 1}$ in $T(Y)$, there exist $n_{k} \rightarrow \infty$ and $\psi \in C(\mathbb{R}, \mathbb{R})$ such that $\lim _{k \rightarrow \infty} \psi_{n_{k}}(\xi)=\psi(\xi)$ uniformly for $\xi$ in any compact subset of $\mathbb{R}$. Since $\phi^{-}(\xi) \leq \psi_{n_{k}}(\xi) \leq \phi^{+}(\xi), \forall \xi \in \mathbb{R}$, we have $\phi^{-}(\xi) \leq \psi(\xi) \leq \phi^{+}(\xi), \forall \xi \in \mathbb{R}$, and hence, $\psi \in Y$. Note that

$$
\lim _{\xi \rightarrow+\infty}\left(\phi^{+}(\xi)-\phi^{-}(\xi)\right) e^{-\lambda \xi}=0
$$

and

$$
\lim _{\xi \rightarrow-\infty}\left(\phi^{+}(\xi)-\phi^{-}(\xi)\right) e^{-\lambda \xi}=0 .
$$

Therefore, for any $\epsilon>0$, there exists $B>0$ such that

$$
0 \leq\left(\phi^{+}(\xi)-\phi^{-}(\xi)\right) e^{-\lambda \xi}<\epsilon, \quad \forall|\xi| \geq B
$$

Since $\lim _{k \rightarrow \infty}\left(\psi_{n_{k}}(\xi)-\psi(\xi)\right) e^{-\lambda \xi}=0$ uniformly for $\xi \in[-B, B]$, there exists an integer $N>0$ such that

$$
\left|\psi_{n_{k}}(\xi)-\psi(\xi)\right| e^{-\lambda \xi}<\epsilon, \quad \forall \xi \in[-B, B], k \geq N .
$$

It then follows that

$$
\left\|\psi_{n_{k}}-\psi\right\|_{\lambda}=\sup _{\xi \in \mathbb{R}}\left|\psi_{n_{k}}(\xi)-\psi(\xi)\right| e^{-\lambda \xi} \leq \epsilon, \quad \forall k \geq N .
$$

This implies that $\lim _{k \rightarrow \infty} \psi_{n_{k}}=\psi$ in $X_{\lambda}$. By the Schauder fixed point theorem, there exists $U \in Y$ such that $U=T(U)$, and hence, $U(x+c n)$ is a traveling wave of (2.3). Since $\phi^{-}(\xi) \leq U(\xi) \leq \phi^{+}(\xi), \forall \xi \in \mathbb{R}$, we have $U(-\infty)=0$ and $U \in \mathcal{C}_{u_{+}^{*}} \backslash\{0\}$. 
Let $u_{n}(x):=U(x+c n), \forall n \geq 0$, and fix a number $\bar{c} \in\left(0, c_{f}^{*}\right)$. By Theorem 2.3 (2), it follows that

$$
0<u_{-}^{*} \leq \liminf _{n \rightarrow \infty,|x| \leq \bar{c} n} u_{n}(x) \leq \limsup _{n \rightarrow \infty,|x| \leq \bar{c} n} u_{n}(x) \leq u_{+}^{*},
$$

and hence,

$$
u_{-}^{*} \leq \liminf _{n \rightarrow \infty} u_{n}(-\gamma n) \leq \limsup _{n \rightarrow \infty} u_{n}(-\gamma n) \leq u_{+}^{*}
$$

uniformly for $\gamma \in[0, \bar{c}]$. This implies that

$$
u_{-}^{*} \leq \liminf _{n \rightarrow \infty} U(s n) \leq \limsup _{n \rightarrow \infty} U(s n) \leq u_{+}^{*}
$$

uniformly for $s \in[c-\bar{c}, c]$. Let

$$
a_{n}=n(c-\bar{c}), \quad b_{n}=n c, \quad \forall n \geq 1 .
$$

Thus, there exists $N_{0}>0$ such that $a_{n+1}-b_{n}<0, \forall n \geq N_{0}$, and hence,

$$
\cup_{n \geq m}\left[a_{n}, b_{n}\right]=\left[a_{m},+\infty\right), \forall m \geq N_{0} .
$$

It then follows that

$$
u_{-}^{*} \leq \liminf _{\xi \rightarrow+\infty} U(\xi) \leq \limsup _{\xi \rightarrow+\infty} U(\xi) \leq u_{+}^{*} .
$$

If, in addition, either (C1) or (C2) holds, then Theorem 2.3 (3) implies that

$$
\lim _{n \rightarrow \infty,|x| \leq \bar{c} n} u_{n}(x)=u^{*}, \forall \bar{c} \in\left(0, c_{f}^{*}\right)
$$

By the same arguments as above, we further have $U(+\infty)=u^{*}$. $\square$

TheOREM 3.2. Let $(F 1)-(F 3)$ hold. Then (2.3) has a traveling wave $U\left(x+c_{f}^{*} n\right)$ such that $U \in \mathcal{C}_{u_{+}^{*}} \backslash\left\{0, u^{*}\right\}$ and

$$
u_{-}^{*} \leq \liminf _{\xi \rightarrow+\infty} U(\xi) \leq \limsup _{\xi \rightarrow+\infty} U(\xi) \leq u_{+}^{*} .
$$

If, in addition, either (C1) or (C2) holds, then $U(+\infty)=u^{*}$.

Proof. Choose a sequence $\left\{c_{j}\right\}_{j \geq 1} \subset\left(c_{f}^{*},+\infty\right)$ such that $\lim _{j \rightarrow \infty} c_{j}=c_{f}^{*}$. By Theorem $3.1(2)$, it follows that $(2.3)$ has a traveling wave $U_{j}\left(x+c_{j} n\right)$ such that $U_{j} \in \mathcal{C}_{u_{+}^{*}} \backslash\{0\}, U_{j}(-\infty)=0$ and

$$
u_{-}^{*} \leq \liminf _{\xi \rightarrow+\infty} U_{j}(\xi) \leq \limsup _{\xi \rightarrow+\infty} U_{j}(\xi) \leq u_{+}^{*} .
$$

Without loss of generality, we assume that $U_{j}(0)=\frac{1}{2} u_{-}^{*}>0, \forall j \geq 1$. Note that

$$
U_{j}(\xi)=\int_{\mathbb{R}} f\left(U_{j}\left(\xi-c_{j}-y\right) k(y) d y, \forall \xi \in \mathbb{R}, j \geq 1 .\right.
$$

It follows that

$$
\left|U_{j}\left(\xi_{1}\right)-U_{j}\left(\xi_{2}\right)\right| \leq b \cdot g\left(\xi_{1}-\xi_{2}\right), \forall \xi_{1}, \xi_{2} \in \mathbb{R}, j \geq 1,
$$


where $g(\xi)$ is defined as in the proof of Theorem 3.1. Then the family of functions $\left\{U_{j}(\xi): j \geq 1\right\}$ is uniformly bounded and equicontinuous in $\xi \in \mathbb{R}$. Thus, there exist $j_{k} \rightarrow+\infty$ and $U \in C(\mathbb{R}, \mathbb{R})$ such that $\lim _{k \rightarrow \infty} U_{j_{k}}(\xi)=U(\xi)$ uniformly for $\xi$ in any compact subset of $\mathbb{R}$. Clearly, $U \in \mathcal{C}_{u_{+}^{*}}$ and $U(0)=\frac{1}{2} u_{-}^{*}$. Letting $j=j_{k} \rightarrow+\infty$ in (3.7) and using the dominated convergence theorem, we obtain

$$
U(\xi)=\int_{\mathbb{R}} f\left(U\left(\xi-c_{f}^{*}-y\right) k(y) d y, \forall \xi \in \mathbb{R},\right.
$$

and hence, $u_{n}(x):=U\left(x+c_{f}^{*} n\right)$ is a traveling wave of (2.3). As in the proof of Theorem 3.1 (2), we see that Theorem 2.3 (2) and (3) imply the asymptotic behavior of $U(\xi)$ as $\xi \rightarrow+\infty$. $\mathrm{C}$

Compared with Theorem 3.1 (2), we expect that $U(-\infty)=0$ in Theorem 3.2. However, we are not able to prove it at this moment since the limiting function $U(\xi)$ may not be nondecreasing on $\mathbb{R}$.

4. Examples. In this section, we present illustrative examples by choosing three types of growth functions from population biology.

First, we consider the logistic type function $f(u)=r u\left(1-\frac{u}{K}\right), r>0, K>0$. Clearly, $f^{\prime}(0)=r, \max _{u \in[0, K]} f(u)=f(K / 2)=\frac{r K}{4}, \frac{f(u)}{u}=r\left(1-\frac{u}{K}\right)$ is strictly decreasing on $(0, K]$, and $u^{*}:=K\left(1-\frac{1}{r}\right)$ is the unique positive fixed point of $f$ on $[0, K]$. Assume that $1<r<4$ so that we have $f^{\prime}(0)>1$ and $f((0, K]) \subset(0, K]$. It is easy to verify that $f(u)$ is strictly increasing on $\left[0, u^{*}\right]$ if $r \in(0,2]$. In the case where $r \in(1,2]$, we choose $b=: u^{*}$, and hence, $u_{-}^{*}=u_{+}^{*}=u^{*}$. In the case $r \in(2,4)$, we choose $b:=\frac{r K}{4}$, and hence, $u_{+}^{*}=b, u_{-}^{*}=f(b)=\frac{r^{2} K(4-r)}{16}$. Note that

$$
\frac{f^{2}(u)}{u}=\frac{r^{2}}{K^{3}}\left(K^{2}(K-u)-r u(K-u)^{2}\right) .
$$

It then follows that $f(u)$ satisfies the property (P2) if $r \in(2,3]$. By Theorems 2.1 and 2.3 and Theorems 3.1 and 3.2, we have the following result.

EXAMPLE 4.1. Let $f(u)=r u\left(1-\frac{u}{K}\right)$ with $K>0$ and $r \in(1,4), b, u_{+}^{*}$ and $u_{-}^{*}$ be defined as above, and $c_{f}^{*}$ be defined as in (2.2) with $h=f$. Then the following statements are valid:

(i) $c_{f}^{*}$ is the spreading speed of (2.3) in the sense that both conclusions (1) and (2) in Theorem 2.3 hold. Further, the conclusion (3) in Theorem 2.3 holds in the case where $r \in(1,3]$.

(ii) For any $c \in\left(0, c_{f}^{*}\right)$, (2.3) has no traveling wave $U(x+c n)$ with $U \in \mathcal{C}_{b} \backslash\{0\}$ and $U(-\infty)=0$, and for any $c \geq c_{f}^{*}$, (2.3) has a traveling wave $U(x+c n)$ with $U \in \mathcal{C}_{u_{+}^{*}} \backslash\left\{0, u^{*}\right\}$ and

$$
u_{-}^{*} \leq \liminf _{\xi \rightarrow+\infty} U(\xi) \leq \limsup _{\xi \rightarrow+\infty} U(\xi) \leq u_{+}^{*} .
$$

Further, $U(+\infty)=u^{*}$ in the case where $r \in(1,3]$. If $r \in(1,2]$, then $U(-\infty)=0$ and $U(\xi)$ is nondecreasing in $\xi$ for all $c \geq c_{f}^{*}$. If $r \in(2,3]$, then $U(-\infty)=0$ for all $c>c_{f}^{*}$.

In Example 4.1, we can also verify that for any $r>0, u f(u)$ is strictly increasing on $[0,2 K / 3]$ and strictly decreasing on $[2 K / 3,+\infty)$. It then follows that $f(u)$ satisfies the property (P1) if $r \in(2,8 / 3]$, but does not satisfies the property (P1) if $r \in(8 / 3,4)$. So we chose to use the property (P2) to obtain the upward convergence as stated in 
Theorem 2.3 (3) for $r$ in a larger interval $(1,3]$. By taking $u_{0}$ as a constant function in integrodifference equation (2.3), we see that the upward convergence implies that $u^{*}$ is a globally attractive fixed point for the map $f$ on $\left(0, u_{+}^{*}\right]$. Note that $\left|f^{\prime}\left(u^{*}\right)\right|=$ $|2-r|>1, \forall r \in(3,4)$. By [5, Theorem 3.8], it then follows that for any $r \in(3,4)$, $u^{*}$ is a unstable fixed point of $f$. This implies that the upward convergence does not hold for any $r \in(3,4)$. Thus, the interval $(1,3]$ for parameter $r$ is optimal for the upward convergence.

Among other things, Kot [7] observed numerically four types of discrete-time traveling waves for the integrodifference equation (2.3) with $f(u)=\left(1+r_{0}\right) u-r_{0} u^{2}$ and $k(x)=3 e^{-6|x|}$ : a simple monotone traveling for $r_{0}=0.9$ ([7, Figure 7]); a traveling wave with damped spatial oscillations for $r_{0}=1.9$ ([7, Figure 8]); a traveling two-cycle for $r_{0}=2.2$ ([7, Figure 9a,b]; and a traveling four-cycle for $r_{0}=2.5([7$, Figure 10a-d]. Clearly, this system is a special case of Example 4.1 with $r=1+r_{0}$ and $K=\left(1+r_{0}\right) / r_{0}$. It is easy to see that our analytic results in Example 4.1 are consistent with these numerical simulations. Note that there is an increasing sequence of parameter values $r_{1}=3<r_{2} \approx 3.449<r_{3} \approx 3.544<r_{4} \approx 3.564<\ldots$ at which the logistic map $f(u)=r u\left(1-\frac{u}{K}\right)$ repeatedly undergoes a period-doubling bifurcation (see, e.g., [5, Section 3.5]). Further, when $r \approx 3.839, f$ has a unique asymptotically stable periodic orbit of minimal period 3. It is a challenging problem to prove the existence of a traveling three-cycle for the integrodifference equation (2.3) associated with the logistic map when $r \approx 3.839$.

Next, we consider the Ricker type function $f(u)=q u e^{-p u}, q>1, p>0$. Clearly, $f^{\prime}(0)=q$ and $\frac{f(u)}{u}=q e^{-p u}$ is strictly decreasing on $(0,+\infty)$. It is easy to see that $u^{*}:=\frac{\ln q}{p}$ is the unique positive fixed point of $f$ on $[0,+\infty)$, that $\max _{u \in[0,+\infty)} f(u)=$ $f(1 / p)=\frac{q}{p e}$, and that $f(u)$ is strictly increasing on $\left[0, u^{*}\right]$ if $q \in(1, e]$. In the case where $q \in(1, e]$, we choose $b:=u^{*}$, and hence, $u_{-}^{*}=u_{+}^{*}=u^{*}$. In the case where $q>e$, we choose $b:=\frac{q}{p e}$, and hence, $u_{+}^{*}=b, u_{-}^{*}=f(b)=\frac{q^{2}}{p e} e^{-q / e}$. Note that

$$
\frac{f^{2}(u)}{u}=q^{2} e^{-p\left(u+q u e^{-p u}\right)} .
$$

An elementary analysis shows that $f(u)$ satisfies the property (P2) if $q \in\left(e, e^{2}\right]$. By Theorems 2.1 and 2.3 and Theorems 3.1 and 3.2, we have the following result.

EXAMPLE 4.2. Let $f(u)=q u e^{-p u}$ with $q>1$ and $p>0, b, u_{+}^{*}$ and $u_{-}^{*}$ be defined as above, and $c_{f}^{*}$ be defined as in (2.2) with $h=f$. Then the following statements are valid:

(i) $c_{f}^{*}$ is the spreading speed of (2.3) in the sense that both conclusions (1) and (2) in Theorem 2.3 hold. Further, the conclusion (3) in Theorem 2.3 holds in the case where $q \in\left(1, e^{2}\right]$.

(ii) For any $c \in\left(0, c_{f}^{*}\right)$, (2.3) has no traveling wave $U(x+c n)$ with $U \in \mathcal{C}_{b} \backslash\{0\}$ and $U(-\infty)=0$, and for any $c \geq c_{f}^{*}$, (2.3) has a traveling wave $U(x+c n)$ with $U \in \mathcal{C}_{u_{+}^{*}} \backslash\left\{0, u^{*}\right\}$ and

$$
u_{-}^{*} \leq \liminf _{\xi \rightarrow+\infty} U(\xi) \leq \limsup _{\xi \rightarrow+\infty} U(\xi) \leq u_{+}^{*}
$$

Further, $U(+\infty)=u^{*}$ in the case where $q \in\left(1, e^{2}\right]$. If $q \in(1, e]$, then $U(-\infty)=0$ and $U(\xi)$ is nondecreasing in $\xi$ for all $c \geq c_{f}^{*}$. If $q \in\left(e, e^{2}\right]$, then $U(-\infty)=0$ for all $c>c_{f}^{*}$. 
In Example 4.2, for any $q>e^{2}$, we have $\left|f^{\prime}\left(u^{*}\right)\right|=|1-\ln q|>1$, and hence, $u^{*}$ is an unstable fixed ploint of $f$. As discussed in Example 4.1, it follows that the interval $\left(1, e^{2}\right]$ for parameter $q$ is optimal for the upward convergence.

Finally, we consider the generalized Beverton-Holt type function $f(u)=\frac{p u}{q+u^{m}}$, $m>0$, and $p>q>0$. Clearly, $f^{\prime}(0)=p / q$ and $\frac{f(u)}{u}=\frac{p}{q+u^{m}}$ is strictly decreasing on $(0,+\infty)$. It is easy to see that $u^{*}:=(p-q)^{\frac{1}{m}}$ is the unique positive fixed point of $f$ on $[0,+\infty)$, and that $f(u)$ is strictly increasing on $[0,+\infty)$ in the case where $m \in(0,1]$. In the case where $m>1$, we have

$$
\max _{u \in[0,+\infty)} f(u)=f(\bar{u})=\frac{p(m-1) \bar{u}}{q m}, \quad \bar{u}:=\left(\frac{q}{m-1}\right)^{\frac{1}{m}} .
$$

By elementary analysis, it follows that $f(u)$ is strictly increasing on $\left[0, u^{*}\right]$ if $m \in$ $(1, p /(p-q)]$, that $u f(u)$ is strictly increasing on $[0,+\infty)$ if $m \in(0,2]$, that $u f(u)$ is strictly increasing on $\left[0,(2 q /(m-2))^{\frac{1}{m}}\right]$ if $m>2$. Define $b:=u^{*}$ if $m \in(0, p /(p-q)]$ and $b:=f(\bar{u})$ if $m>p /(p-q)$. It then follows that $u_{-}^{*}=u_{+}^{*}=u^{*}$ in the case where $m \in(1, p /(p-q)]$, and

$$
u_{+}^{*}=b, \quad u_{-}^{*}=f(b)=\frac{p^{2}(m-1) \bar{u}}{q^{2} m+\frac{p^{m}(m-1)^{m-1} q}{(q m)^{m-1}}},
$$

in the case where $m>p /(p-q)$. Note that $f(u)$ satisfies the property (P1) if either $m \in(0,2]$, or $m>\max (2, p /(p-q))$ and $f(\bar{u}) \leq(2 q /(m-2))^{\frac{1}{m}}$. By Theorems 2.1 and 2.3 and Theorems 3.1 and 3.2, we have the following result.

EXAMPLE 4.3. Let $f(u)=\frac{p u}{q+u^{m}}$ with $m>0$ and $p>q>0, b, u_{+}^{*}$ and $u_{-}^{*}$ be defined as above, and $c_{f}^{*}$ be defined as in (2.2) with $h=f$. Then the following statements are valid:

(i) $c_{f}^{*}$ is the spreading speed of (2.3) in the sense that both conclusions (1) and (2) in Theorem 2.3 hold. Further, the conclusion (3) in Theorem 2.3 holds in the case where either $m \in(0, \max (2, p /(p-q))]$, or $m>\max (2, p /(p-q))$ and $f(\bar{u}) \leq(2 q /(m-2))^{\frac{1}{m}}$.

(ii) For any $c \in\left(0, c_{f}^{*}\right)$, (2.3) has no traveling wave $U(x+c n)$ with $U \in \mathcal{C}_{b} \backslash\{0\}$ and $U(-\infty)=0$, and for any $c \geq c_{f}^{*}$, (2.3) has a traveling wave $U(x+c n)$ with $U \in \mathcal{C}_{u_{+}^{*}} \backslash\left\{0, u^{*}\right\}$ and

$$
u_{-}^{*} \leq \liminf _{\xi \rightarrow+\infty} U(\xi) \leq \limsup _{\xi \rightarrow+\infty} U(\xi) \leq u_{+}^{*} .
$$

Further, $U(+\infty)=u^{*}$ in the case where either $m \in(0, \max (2, p /(p-q))]$, or $m>\max (2, p /(p-q))$ and $f(\bar{u}) \leq(2 q /(m-2))^{\frac{1}{m}}$. If $m \in(0, p /(p-q)]$, then $U(-\infty)=0$ and $U(\xi)$ is nondecreasing in $\xi$ for all $c \geq c_{f}^{*}$. If either $p /(p-q)<m \leq 2$, or $m>\max (2, p /(p-q))$ and $f(\bar{u}) \leq(2 q /(m-2))^{\frac{1}{m}}$, then $U(-\infty)=0$ for all $c>c_{f}^{*}$.

Acknowledgments. We are grateful to two anonymous referees for their careful reading and helpful suggestions which led to an improvement of our original manuscript. Xiao-Qiang Zhao would like to thank the National Center for Theoretical Science, Tsing Hua University, Taiwan for its kind hospitality during his visit there. 


\section{REFERENCES}

[1] D. G. Aronson and H. F. Weinberger, Nonlinear diffusion in population genetics, combustion, and nerve pulse propagation, in Partial Differential Equations and Related Topics, J. A. Goldstein, ed., Lecture Notes in Mathematics Ser., 446. Springer-Verlag, 1975, pp. 5-49.

[2] D. G. Aronson And H. F. WeinBerger, Multidimensional nonlinear diffusion arising in population dynamics, Adv. Math., 30 (1978), pp. 33-76.

[3] O. Diekmann, Thresholds and traveling waves for the geographical spread of infection, J. Math. Biol., 6 (1978), pp. 109-130.

[4] T. FARIA, W. HuAng and J. Wu, Traveling waves for delayed reaction-diffusion equations with global response, Proc. Roy. Soc. Lon., Ser A., 462 (2006), pp. 229-261.

[5] J. K. Hale and H. Kocak, Dynamics and Bifurcations, Springer-Verlag, New York, 1991.

[6] A. Hastings, K. Cuddington, K.F. Davies, C. J. Dugaw, S. Elmendorf, A. Freestone, S. Harrison, M. Holland, J. Lambrinos, U. Malvadkar, B. A. Melbourne, K. Moore, C. TAYlor And D. Thomson, The spatial spread of invasions: new developments in theory and evidence, Ecology Letters, 8 (2005), pp. 91-101.

[7] M. Кот, Discrete-time traveling waves: ecological examples, J. Math. Biology, 30 (1992), pp. 413-436.

[8] J. M. Levine, E. Pachepsky, B. E. Kendall, S. G. Yelenik and J. H. R. Lambers, Plant-soil feedbacks and invasive spread, Ecology Letters, 9 (2006), pp. 1005-1014.

[9] M. Кот, M. A. Lewis And P. VAn Den Driessche, Dispersal data and the spread of invading organisms, Ecology, 77 (1996), pp. 2027-2042.

[10] B. Li, H. F. Weinberger And M. A. Lewis, Spreading speeds as slowest wave speeds for cooperative systems, Math. Biosci., 196 (2005), pp. 82-89.

[11] X. Liang AND X.-Q. ZhaO, Asymptotic speeds of spread and traveling waves for monotone semiflows with applications, Communications on Pure and Appl. Math., 60 (2007), pp. 1-40. Erratum: 61 (2008), pp. 137-138.

[12] X. Liang, Y. Yi And X.-Q. ZhaO, Spreading speeds and traveling waves for periodic evolution systems, J. Differential Equations, 231 (2006), pp. 57-77.

[13] R. LuI, Biological growth and spread modeled by systems of recursions, I. Mathematical theory, Math. Biosci., 93 (1989), pp. 269-295.

[14] S. MA, Traveling waves for non-local delayed diffusion equations via auxiliary equations, J. Differential Equations, 237 (2007), pp. 259-277.

[15] M. Neubert and H. Caswell, Demography and dispersal: calculation and sensitivity analysis of invasion speed for structured populations, Ecology, 81 (2000), pp. 1613-1628.

[16] C. Ou AND J. Wu, Persistence of wavefronts in delayed nonlocal reaction-diffusion equations, J. Differential Equations, 235 (2007), pp. 219-261.

[17] H. R. Thieme, Density-dependent regulation of spatially distributed populations and their asymptotic speed of spread, J. Math. Biology, 8 (1979), pp. 173-187.

[18] H. R. Thieme, On a class of Hammerstein integral equations, Manuscripta Math., 29 (1979), pp. $49-84$

[19] H. R. Thieme And X.-Q. Zhao, Asymptotic speeds of spread and traveling waves for integral equations and delayed reaction-diffusion models, J. Differential Equations, 195 (2003), pp. 430-470.

[20] H. F. WeinBerger, Long-time behavior of a class of biological models, SIAM J. Math. Anal., 13 (1982), pp. 353-396.

[21] H. F. Weinberger, M. A. Lewis And B. Li, Analysis of linear determinacy for spread in cooperative models, J. Math. Biol., 45 (2002), pp. 183-218.

[22] J. Wu AND X. Zou, Traveling wave fronts of reaction-diffusion systems with delay, J. Dynamics and Differential Equations, 13 (2001), pp. 651-687. 